\title{
Correlated few-particle states in artificial bipolar molecule
}

\author{
Egidijus Anisimovas * and F. M. Peeters \\ Departement Natuurkunde, Universiteit Antwerpen (UIA), B-2610 Antwerpen, Belgium
}

(Dated: 22 January 2002)

\begin{abstract}
We investigate the ground and excited states of a bipolar artificial molecule composed of two vertically coupled quantum dots containing different type of carriers - electrons and holes - in equilibrium. The approach based on exact diagonalization is used and reveals an intricate pattern of ground-state angular momentum switching and a rearrangement of approximate single-particle levels as a function of the inter-dot coupling strength.
\end{abstract}

PACS numbers: 73.21.La

In recent decades, artificially created zero-dimensional systems - the so-called quantum dots or artificial atoms - have been a major focus of active research in condensed matter physics [1]. Most of the interest arises from our ability to control the addition or extraction of even a single particle [2, 3] as well as from the fact that these artificial structures often offer a rich set of novel features unrivaled by those of their natural counterparts. For example, while the nature of atomic spectra is mostly governed by the quantization of motion of a single particle in the field of the nucleus, in quantum dots the crucial role of the quantizing magnetic field and strong Coulomb interaction between the electrons leads to the formation of intriguing 'magic' states [1, \# related to the compact states of non-interacting composite fermions [1], 5].

The ability to create and study double-layer systems has added a new dimension to the physics of quantum dots. Two vertically stacked quantum dots make an example of an artificial molecule. The observation of a novel incompressible state induced by the inter-layer coupling [6] motivated a study of correlated few-particle states in vertical double-dot systems [7]. In this work, the interdot interactions were analysed in terms of an isospin$1 / 2$, and a new class of incompressible ground states was found.

In the present paper, we examine the correlated fewparticle states of an artificial bipolar molecule consisting of two vertically stacked quantum dots populated by carriers of different kinds - electrons and holes, respectively. Such a system could be structured, for example, in bilayer-bipolar heterostructures containing electron and hole layers in equilibrium. These structures have been realized in the crossed gap InAs/GaSb system \& as well as in biased GaAs/AlGaAs heterostructure [9, 10] where electron and hole layers form on the opposite sides of an AlGaAs barrier. Most of the interest in such systems stems from the possibility (at least, in principle) of the formation of Bose-Einstein condensate of indirect excitons 11].

While the superfluid state has not been demonstrated so far, a number of other interesting effects of electronhole coupling and hybridization have been predicted and observed. For example, the Coulomb-drag experiments [10. 12] revealed the inter-layer momentum transfer rates up to an order of magnitude larger than in similar electron-electron systems, and magnetotransport calculations [13] established the presence of coupling-dependent current that remains large even when the Fermi energy lies in the hybridization gap.

In the present paper, we consider two vertically coupled infinitesimally thin circularly-symmetric quantum dots placed into a strong perpendicular magnetic field. The confining potentials of the dots are assumed to be of a simple parabolic shape, and we choose the base frequencies of the two dots to be inversely proportional to the effective masses of the respective carriers, i.e. $\omega_{e} m_{e}=\omega_{h} m_{h}$. This choice implies the equality of the quantum dot oscillator lengths $l_{0}=\sqrt{\hbar / m_{a} \omega_{a}}$ for $a=e, h$ (a plausible assumption) as well as the equality of renormalized oscillator lengths in magnetic fields of arbitrary strength since $l^{-4}=l_{0}^{-4}+(1 / 4) l_{c}^{-4}$ and the cyclotron radius $l_{c}=\sqrt{\hbar c / e B}$ does not depend on the material parameters. The above constraint also considerably simplifies the calculation of Coulomb matrix elements between the electron and hole states (see below).

In the limit of strong magnetic fields, when the cyclotron frequencies of the two types of carriers $\omega_{c(e)}$ and $\omega_{c(h)}$ exceed the respective base dot frequencies (in practice, $B>2-3 \mathrm{~T}$ ) we can restrict the single-particle bases in the dots to the essentially one-dimensional sets of the lowest-Landau-level states. The energies of these states are spaced equidistantly by $\varepsilon_{a}=\hbar \omega_{a}^{2} / \omega_{c(a)}$ with $a=e, h$. We note that due to the inverse proportionality of the cyclotron frequency to the effective mass we have $\varepsilon_{e} / \varepsilon_{h}=m_{h} / m_{e}$.

These states are labelled by a single index $n=0,1,2 \ldots$

$$
\psi_{n}(r, \theta)=\frac{1}{\sqrt{\pi n !}} \mathrm{e}^{\mp i n \theta} \frac{r^{n}}{l^{n+1}} \mathrm{e}^{-r^{2} / 2 l^{2}},
$$

where the upper sign is to be used for electrons and the lower one for holes. That is, the electrons can only have negative or zero angular momenta and vice versa. Moreover, since the particles in strong magnetic fields are spinpolarized we can neglect the presence of the spin degree 
of freedom and also drop the constant Zeeman energy.

For a many particle system, a convenient basis is given by the set of all possible Slater determinants (equivalently, ordered strings of the associated creation operators) composed of the functions (11). Thus we will denote possible configurations of the molecule by enumerating the single-particle quantum numbers $n_{i}$ in ascending order, first for electrons and then, separated by a semicolon, for holes. E.g. the ground state of three non-interacting electrons and holes is given by $\{012 ; 012\}$. Due to the cylindrical symmetry of the molecule the many-body states of different total angular momenta $M_{t}$ will not be mixed, thus we will be able to perform diagonalizations separately in subspaces of a given $M_{t}$. As a matter of fact, in our basis the single-body part of the Hamiltonian is diagonal and the remaining task is the calculation and diagonalization of the Coulomb interactions.

Before proceeding any further, it is convenient to introduce handy dimensionless units thus also clarifying the relevant energy and length scales of the problem. From now on we will measure all the energies in $\varepsilon_{e}$ and the lengths in $l$. Then the spacing of the electronic singleparticle is set equal to 1 , and the spacing of the hole levels is $m_{e} / m_{h}$. The interaction part of the Hamiltonian becomes

$$
\begin{aligned}
V= & \lambda\left\{\sum_{i>j}^{N_{e}} \frac{1}{\left|\mathbf{r}_{i}-\mathbf{r}_{j}\right|}+\sum_{i>j}^{N_{h}} \frac{1}{\left|\mathbf{r}_{i}-\mathbf{r}_{j}\right|}\right. \\
& \left.-\sum_{i}^{N_{e}} \sum_{j}^{N_{h}} \frac{1}{\left|\mathbf{r}_{i}-\mathbf{r}_{j}+\vec{\Delta}\right|}\right\} .
\end{aligned}
$$

Here, $\vec{\Delta}$ represents the vertical separation between the two dots, and

$$
\lambda=\frac{l_{0}^{4}}{a_{B}^{*} l_{c}^{2} l} \approx \frac{l_{0}^{4}}{\sqrt{2} a_{B}^{*} l_{c}^{3}} \sim B^{3 / 2}
$$

expresses the relative strength of inter-particle interactions in terms of a ratio of the characteristic lengths, $a_{B}^{*}$ being the effective electronic Bohr radius.

We calculate the matrix elements of the Coulomb interaction between various many-body states by decomposing them into a sum of two-particle Coulomb integrals

$$
J=\int d^{2} r_{1} \int d^{2} r_{2} \psi_{n_{1}}^{*}\left(\mathbf{r}_{1}\right) \psi_{n_{2}}^{*}\left(\mathbf{r}_{2}\right) v \psi_{n_{3}}\left(\mathbf{r}_{1}\right) \psi_{n_{4}}\left(\mathbf{r}_{2}\right)
$$

with $v=\left|\mathbf{r}_{1}-\mathbf{r}_{2}\right|^{-1}$ if both particles belong to the same layer, and $v=\left|\mathbf{r}_{1}-\mathbf{r}_{2}+\vec{\Delta}\right|^{-1}$ otherwise. In the former case the integral is easily evaluated analytically using the complex-variable technique due to Girvin and Jach [14] to separate the CM and relative coordinates (see formula (7) in their paper). When the particles $\mathbf{r}_{1}$ and $\mathbf{r}_{2}$ belong to different layers the only modification is the replacement $\rho^{-1} \rightarrow\left(\rho^{2}+\Delta^{2}\right)^{-1 / 2}$ in the integral over the radial part

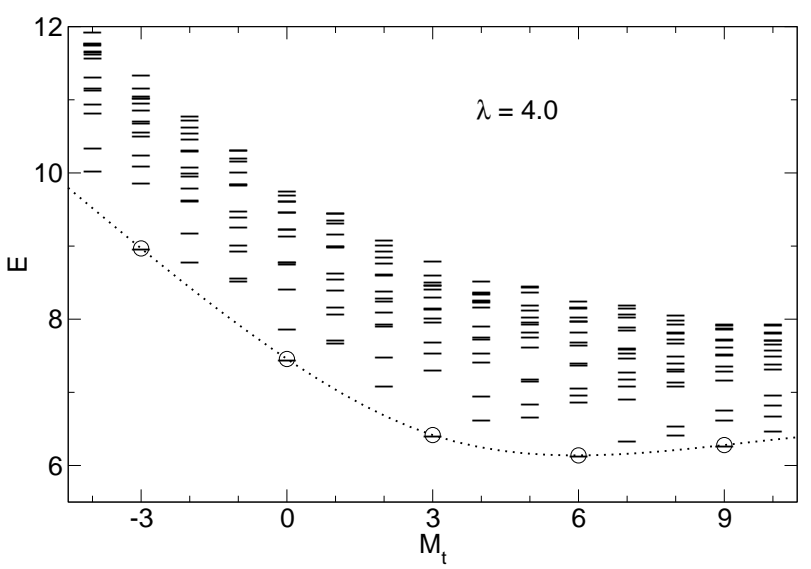

FIG. 1: Angular-momentum resolved spectrum of an artificial molecule in the weak-coupling limit. The stable low-energy 'magic' states are marked by open dots and joined by a dashed line.

of the relative coordinate $\rho$ which has to be computed numerically.

We numerically calculated energy levels and manyparticle states of neutral systems consisting of equal numbers (1, 2 and 3$)$ of electrons and holes in the dots. However, in order to keep the presentation concise, we will concentrate on the most illustrative results obtained for a six-particle artificial molecule. Systems involving smaller number of particles show qualitatively similar behaviour.

Weakly correlated dots. When the distance between the two dots is relatively large - such as $\Delta=3.3$ (in the units $l$ ) to be used in the present example - the interdot interactions between the particles are rather weak compared to the strong Coulomb repulsion within the individual dots which is decisive in the determination of the overall behaviour of the molecule. In Fig. 11 we plot the angular-momentum resolved spectrum of the system showing 15 lowest states for each value of $M_{t}$ at $\lambda=4.0$. One sees that for the values of $M_{t}$ equal to multiples of 3 the lowest-energy states (marked by the open circles) are typically considerably lower than those corresponding to the neighbouring values of the angular momentum. This is a consequence of the fact that the individual dots 'prefer' to be in the well-known 'magic' states. For three particles (electrons or holes) the predominant single-particle configurations in these stable many-body states are $\{012\},\{123\},\{234\}$, etc. It will be instructive to compare these configurations with their counterparts observed in strongly coupled dots below.

Since the effective mass of the holes is considerably larger than that of electrons (we use $m_{h}=6.7 m_{e}$ ) the Coulomb repulsion promotes them to states of higher angular momentum more easily. In Fig. 1 we see that the ground state has $M_{t}=6$, and the dashed lowest-energy curve rises much more sharply in the direction of negative angular momenta. 


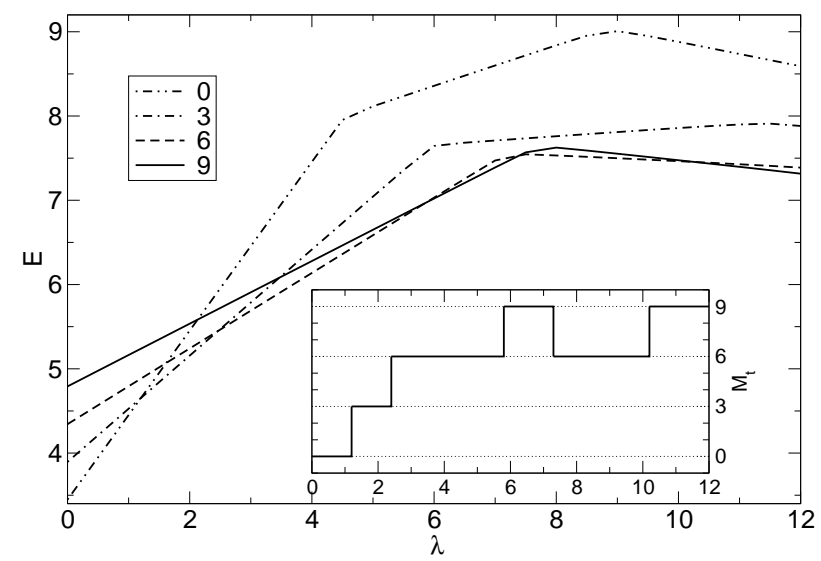

FIG. 2: The dependence of relative ground-state energies with $M_{t}=0,3,6,9$ on the interaction strength $\lambda$. The inset shows the switching of the angular momentum of the absolute ground-state.

While the angular momentum of the ground state is generally expected to grow with increasing Coulomb repulsion, this growth is not monotonous. The inset of Fig. 2 shows the evolution of the ground-state $M_{t}$ versus $\lambda$. Notice that the angular momentum reaches the value $M_{t}=9$ in three monotonous steps $\Delta M_{t}=3$, and then switches back and forth to the value $M_{t}=6$. To understand this kind of behaviour we also investigated the evolution of the relative ground-states (i.e. lowestenergy states of a given $M_{t}$ ) of the involved angular momenta in Fig. 2. We see that the $\lambda$-dependences of these energies typically consist of segments of nearly straight lines connected by abrupt breaks associated with the change of the nature of the states. For example, we observe two breaks in the $M_{t}=0$ curve at $\lambda \approx 4$ and $\lambda \approx 9$. At $\lambda \approx 4$ the molecule minimizes its energy by switching from the dominant configuration $\{012 ; 012\}$ into $\{123 ; 123\}$ followed by the changeover into $\{234 ; 234\}$ at $\lambda \approx 9$. The curves corresponding to higher values of $M_{t}$ also show similar behaviour which in this particular case results in a close competition between the $M_{t}=6$ and $M_{t}=9$ lowest-energy states and switching of the absolute ground-state in a rather extended range of $\lambda$ values.

Strongly correlated dots. Bringing the two dots closer (we will consider the case of $\Delta=0.2$ ) results in an entirely different character of their energy spectra. The Coulomb attraction between particles of different kind wins over the intra-dot repulsion and leads to the formation of rather strongly bound and weakly interacting excitons. The energies of the relative ground-states of given angular momenta decrease rapidly and monotonously with increasing $\lambda$ and no longer contain abrupt breaks. The absolute ground state always has the total angular momentum $M_{t}=0$. For the clarity of presentation, in Fig. 3 we plot the differences of the lowest-energy states

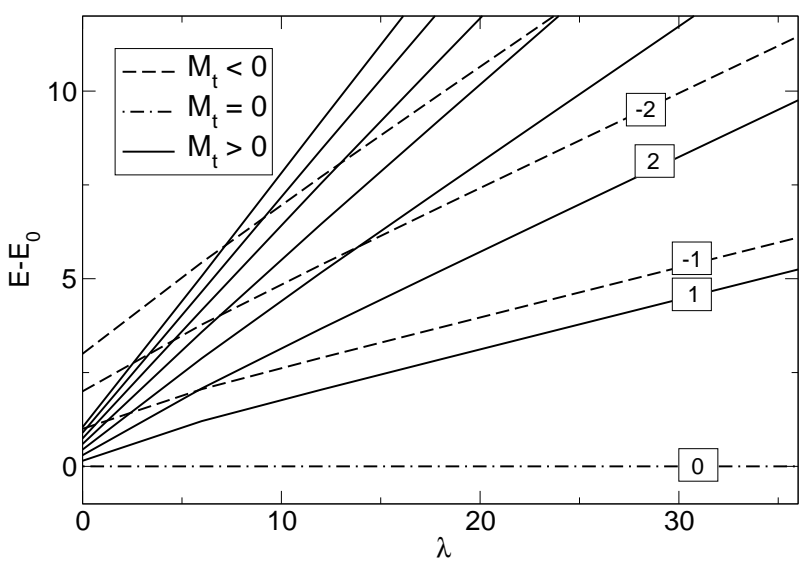

FIG. 3: The evolution of the relative ground-states with $-3 \leq$ $M_{t} \leq 7$ versus the coupling strength $\lambda$. The ground state energy is subtracted from all the curves.

of $M_{t}=-3 \ldots 7$ and the absolute ground state $\left(M_{t}=0\right)$. In this figure we see a clear rearrangement of the structure of the energy levels from the weakly interacting limit to the strongly-interacting limit (high $\lambda$ ). At $\lambda \rightarrow 0$ the levels corresponding to the positive momenta are spaced more closely that the negative-momentum levels due to the higher hole mass. For sufficiently high $\lambda$ values the energy levels regroup so that the levels corresponding to the angular momenta $M_{t}$ and $-M_{t}$ form closely spaced pairs.

A very interesting feature of the correlated electron and hole states in this regime can be observed by looking more closely at the dominant single particle configurations of the many-body states. In the table 1 we compare the configurations corresponding to the relative ground states for $M_{t}=0 \ldots 7$ in the weakly and strongly coupled dots. Since we are looking at the positive momentum states the electronic subsystem is in the energetically most favourable state $\{012\}$ of the total momentum $M_{e}=-3$ while the holes contribute the angular momentum $M_{h}=M_{t}+3$. However, we see that the pattern of filling of the hole states is very distinct in the two regimes. In the weak coupling limit, the holes favour the 'traditional magic' filling scheme in which they occupy a compact set of adjacent angular momentum orbitals, such as $\{123\}$ in the case of $M_{h}=6$. In contrast, when the holes are in the $M_{h}=6$ states in the strong coupling limit, the configuration $\{123\}$ becomes the least favoured one. Instead, the dominant configuration is $\{015\}$ in which two of the holes occupy the orbitals of the lowest possible angular momentum while the third one is placed into a remote orbital $n=5$.

This new type of systematics of the single-particle configurations can be understood by realising that singling out one particle into a remote high- $n$ orbital becomes energetically favourable in the case when the spacing be- 
TABLE I: The predominant single-particle configurations of the relative ground states.

\begin{tabular}{ccc}
\hline \hline$M_{t}$ & Weak inter-dot coupling & Strong inter-dot coupling \\
\hline 0 & $\{012 ; 012\}$ & $\{012 ; 012\}$ \\
1 & $\{012 ; 013\}$ & $\{012 ; 013\}$ \\
2 & $\{012 ; 023\}$ & $\{012 ; 014\}$ \\
3 & $\{012 ; 123\}$ & $\{012 ; 015\}$ \\
4 & $\{012 ; 124\}$ & $\{012 ; 016\}$ \\
5 & $\{012 ; 134\}$ & $\{012 ; 017\}$ \\
6 & $\{012 ; 234\}$ & $\{012 ; 018\}$ \\
7 & $\{012 ; 235\}$ & $\{012 ; 019\}$ \\
\hline \hline
\end{tabular}

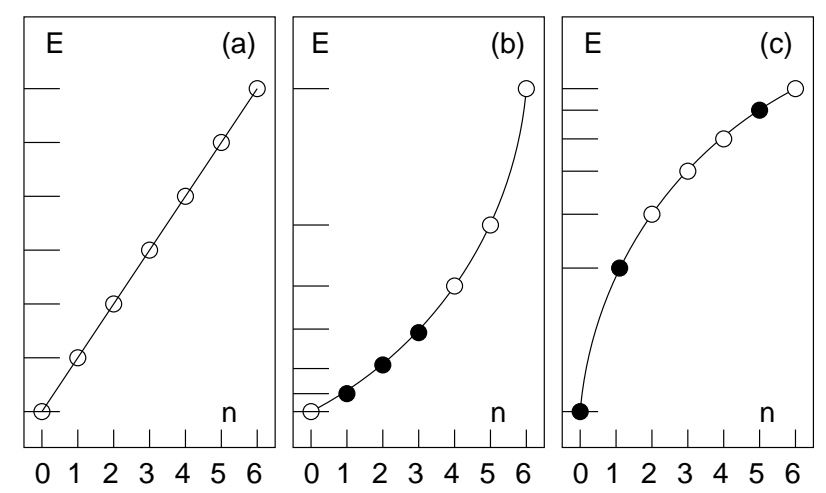

FIG. 4: A schematic plot of single-particle energies versus angular momentum. Panel (a) displays the case of equidistant level spacing, (b) corresponds to the case of energy levels condensing downwards, and (c) represents the case of upwardscondensing energy levels. Closed dots in panels (b) and (c) depict the lowest-energy configurations of total momentum $M_{h}=6$.

tween energy levels is decreasing with increasing $n$, while in the opposite case the preferred configuration is the compact one. We illustrate this point in Fig. 4. Panel (a) shows the dependence of the single-particle energy on its angular momentum for non-interacting particles in a quantum dot. Since the energy levels are equidistant, all three ways of accommodating three particles of the total momentum $M_{h}=6-\{015\},\{024\}$ and $\{123\}-$ have equal total energies. However, if the energy levels are condensing downwards [panel (b)], the preferred configuration is the compact $\{123\}$ one as we observe in the weak-coupling limit. On the other hand, if the energy levels are condensing upwards, as shown in panel (c), the favoured configuration is $\{015\}$ thus reproducing our result in the strong-coupling limit.

In conclusion, we have investigated the many-body states of a bipolar artificial molecule consisting of two vertically aligned quantum dots in two distinct regimes. In the weak-coupling limit we found an intricate pattern of switching of the ground state angular momentum as a function of a perpendicular magnetic field, while in the opposite strong-coupling limit we observe the formation of a new class of energetically favourable states.

This work is supported by the Flemish Science Foundation (FWO-Vl) and the University of Antwerp through a VIS-project. EA is supported by a Marie Curie Fellowship of the EU.

* Electronic address: egidijus@uia.ua.ac.be

$\dagger$ Electronic address: peeters@uia.ua.ac.be

[1] L. Jacak, P. Hawrylak, and A. Wójs, Quantum Dots, (Springer, Berlin, 1998).

[2] S. Tarucha et al., Phys. Rev. Lett. 77, 3613 (1996).

[3] P. Hawrylak, G. A. Narvaez, M. Bayer, and A. Forchel, Phys. Rev. Lett. 85, 389 (2000).

[4] P. A. Maksym and T. Chakraborty, Phys. Rev. Lett. 65, 108 (1990); R. B. Laughlin, Phys. Rev. B 27, 3383 (1983).

[5] J. K. Jain and T. Kawamura, Europhys. Lett. 29, 321 (1995); R. K. Kamilla and J. K. Jain, Phys. Rev. B 52, 2798 (1995).

[6] S. Q. Murphy et al., Phys. Rev. Lett. 72, 728 (1994).

[7] J. J. Palacios and P. Hawrylak, Phys. Rev. B 51, 1769 (1995); see also B. Partoens and F. M. Peeters, Phys. Rev. Lett. 84, 4433 (2000).

[8] See, for example, T. P. Marlow et al., Phys. Rev. Lett. 82, 2362 (1999); J. Kono et al., Phys. Rev. B 55, 1617 (1997); M. Altarelli, Phys. Rev. B 28, 842 (1983) and references therein.

[9] Yu. E. Lozovik and V. I. Yudson, Pis'ma Zh. Eksp. Teor. Fiz. 22, 556 (1975) [JETP Lett. 22, 274 (1975)].

[10] U. Sivan, P. M. Solomon, and H. Shtrikman, Phys. Rev. Lett. 68, 1196 (1992).

[11] Yu. E. Lozovik, O. L. Berman, and V. G. Tsvetus, Phys. Rev. B 59, 5627 (1999).

[12] H. C. Tso, P. Vasilopoulos, and F. M. Peeters, Phys. Rev. Lett. 70, 2146 (1993); L. Świerkowski, J. Szymański, and Z. W. Gortel, Phys. Rev. Lett. 74, 3245 (1995).

[13] Y. Naveh and B. Laikhtman, Europhys. Lett. 55, 545 (2001).

[14] S. M. Girvin and T. Jach, Phys. Rev. B 28, 4506 (1983). 